\title{
Efficacy and safety of co-administration of resveratrol with meloxicam in patients with knee osteoarthritis: a pilot interventional study
}

This article was published in the following Dove Press journal: Clinical Interventions in Aging

\author{
Saad Abdulrahman Hussain' \\ Bushra Hassan Marouf ${ }^{2}$ \\ Ziyad Serdar Ali ${ }^{3}$ \\ Runj Simko Ahmmad' \\ 'Department of Pharmacology and \\ Toxicology, Faculty of Pharmacy, \\ Al-Rafidain University College, \\ Al-Rafidain, Baghdad, Iraq; \\ ${ }^{2}$ Department of Pharmacology and \\ Toxicology, College of Pharmacy, \\ University of Sulaimani, Sulaimani, \\ Kurdistan, Iraq; ${ }^{3}$ Department of \\ Rheumatology and Orthopedics, \\ Shar Teaching Hospital, Sulaimani, \\ Kurdistan, Iraq
}

Background and aim: Resveratrol shows remarkable anti-inflammatory activities in experimental models. This study aims to evaluate the effect of resveratrol, as an adjuvant with meloxicam (Mlx), on the pain and functional activity during a 90-day period and monitor the adverse effects on kidney and liver functions, lipid profile, and hematological markers.

Patients and methods: This study was a double-blind, placebo-controlled, randomized multi-center study that involved 110 patients with knee osteoarthritis (OA) and was performed at Sulaimani City, Iraq, from December 2016 to September 2017. To assess the effects of Mlx with or without resveratrol, pain severity and functional disability were evaluated at baseline and after 90 days using the Western Ontario and McMaster Universities Osteoarthritis Index. Fasting blood was collected to evaluate the lipid profile markers, hematological picture, and liver and kidney functions, in addition to vitamin D level.

Results: Resveratrol significantly improves pain, functions, and associated symptoms compared with placebo. The clinical and biochemical markers indicated that $500 \mathrm{mg} /$ day of resveratrol, as an adjuvant with Mlx, is safe and well tolerated by the knee OA patients.

Conclusion: Resveratrol, as an "add-on" medication with Mlx, was superior in terms of safety and efficacy to Mlx alone for the treatment of pain and improvement of physical function in patients with knee OA.

Keywords: resveratrol, meloxicam, knee OA, WOMAC, alternative medicine, OA pain

\section{Introduction}

Osteoarthritis (OA) is the most common form of inflammatory joint diseases, associated with severe pain, stiffness, limitation of joint movement, and disability. At the cellular level, OA is characterized by a loss of tissue cellularity and damage of the extracellular matrix. ${ }^{1}$ It is recently considered as an inflammatory joint disorder, with chronic and painful inflammatory changes that are associated with physical impairment and chronic disability. ${ }^{2}$ The currently available pharmacological interventions to alleviate inflammation and pain are accompanied by many adverse effects that limit their use as a long-term therapy for OA. Despite recent advances in the understanding of the cellular and molecular processes involved in OA pathogenesis, optimal therapy is still lacking. In this context, there is a growing interest in developing therapeutic modalities capable of relieving the symptomatic inflammation and $\mathrm{OA}$-associated pain with less adverse effect and more tolerability. ${ }^{3}$ Numerous studies have recently highlighted the potential role of natural compounds, including nutraceuticals, in improving symptoms and function in the treatment of OA. ${ }^{4-6}$ Nutraceuticals are good candidates for the management of $\mathrm{OA}$, due to their safety profile and potential efficacy. ${ }^{7}$ Resveratrol
Correspondence: Saad Abdulrahman Hussain

Department of Pharmacology and Toxicology, Faculty of Pharmacy, Al-Rafidain University College, 10052 Al-Mustansiriya, Q. 506, St. 75, Baghdad, Iraq

Tel +964 790l 712624

Email saad.hussain@coalrafidain.edu.iq $\mathrm{BY}$
hereby accept the Terms. Non-commercial uses of the work are permitted without any further permission from Dove Medical Press Limited, provided the work is properly attributed. For permission hereby accept the Terms. Non-commercial uses of the work are permitted without any further permission from Dove Med 
(3,4',5-trihydroxy trans-stilbene) is a natural polyphenolic compound extracted from various fruits, vegetables, and nuts, including grapes, berries, peanuts, pistachios, and root extracts of the weed Polygonum cuspidatum. ${ }^{8,9}$ It has been widely reported that resveratrol has anti-proliferative, anti-oxidative, anti-inflammatory, and analgesic effects in many experimental models. ${ }^{10-14}$ Preclinical studies provided accumulating evidence on its efficacy in ameliorating the degenerative articular damage that mostly attributed to its pleiotropic properties. ${ }^{15,16}$ Moreover, many pieces of evidence have displayed resveratrol as one of the nutraceutical candidates for OA therapy in human. ${ }^{17,18}$ Early reports showed that resveratrol has anti-inflammatory activity in acute and chronic phases of inflammation and that the effect was associated with its ability to modulate cyclooxygenase COX-1 and COX-2. ${ }^{19}$ The safety and tolerability of resveratrol have been studied in many pharmacokinetics and preclinical studies and various doses can be used for different durations; ${ }^{20,21}$ however, no data indicating the safety of resveratrol on lipidhematological profile, liver, and kidney function is currently available. Therefore, this study was designed to evaluate the effect of resveratrol on the pain and functional activity during a 90-day period in knee $\mathrm{OA}$ and to monitor any adverse effects related to kidney and liver functions, lipid profile, and hematological markers.

\section{Patients and methods Study design and ethical consideration}

This was a randomized, double-blind, and placebo-controlled clinical trial with a 3-month follow-up pattern. The study protocol was approved by the local committee of Clinical Research of the College of Medicine, University of Sulaimani (Certificate ID: 42 on 21/11/2016). The trial was conducted in accordance with the Declaration of Helsinki and its amendments and the Guidelines for Good Clinical Practices issued by the Committee of Propriety Medicinal Products of the European Union. It was conducted as a joint project between the University of Sulaimani and Shar Teaching Hospital, General Teaching Hospital and the Rheumatology and Physical Rehabilitation Center in Sulaimani city.

\section{Patient enrollment and consent}

One hundred and twenty-four patients with painful knee OA who were admitted to the Outpatient Department (OPD) of Shar Teaching Hospital, General Teaching Hospital, and Rheumatology and Physical Rehabilitation Center were screened for eligibility, and only 110 patients were found to be eligible and enrolled in the study. All candidates have gone through a standardized interview process and received detailed information about the study protocol and the treatment intervention. Following the interview, written informed consents were obtained from each participant. The participants have the right to withdraw from the study at any time for any reason without consequences. The trial was executed from December 2016 to November 2017, including enrollment and follow-up (Figure 1).

\section{Inclusion and exclusion criteria}

The eligibility criteria for enrollment were based on the clinical diagnosis of knee OA according to the updated guidelines of the American College of Rheumatology, 2012. ${ }^{22}$ OA was graded according to the radiographic finding of the affected knee and based on the Kellgren and Lawrence grading system ${ }^{23}$ as follows: grade 0 , no radiological changes; grade 1, doubtful narrowing of joint space and possible osteophytic lipping; grade 2, definite osteophytes and possible narrowing of joint space; and grade 3, moderate multiple osteophytes, definite narrowing of joint space, some sclerosis, and possible deformity of bone contours. ${ }^{24}$ In this study, patients were required to have Kellgren and Lawrence grade of 1-3 in at least one knee. The study also includes both genders between 45 and 75 years of age with patient assessment of OA pain in Western Ontario and McMaster Universities Osteoarthritis Index (WOMAC) above 50 out of 96 scores at baseline, and duration of pain of at least 6 months on the majority of days during the preceding months. The patients were required to be chronic users of nonsteroidal antiinflammatory drugs and/or acetaminophen for OA pain. The exclusion criteria include patients with concomitant medical or arthritic conditions confounding evaluation of the knee OA, predominant symptomatic patellofemoral OA or history of a clinically significant trauma/surgery to the affected knee, grade 4 OA on the Kellgren-Lawrence grading system, severe cardiovascular, lung, liver, and kidney diseases, and cancer, and patients taking coumarin-type anticoagulants, heparins, and anti-platelets such as clopidogrel or glucocorticoid treatment within the previous 4 weeks. Patients using supplements with antioxidants that contain polyphenols were also excluded. The participants were not allowed to use any other analgesics or anti-inflammatory agents during the study.

\section{Intervention}

The eligible participants were randomly assigned to one of the two treatment groups using a simple randomization sequence of 1:1 ratio. The investigators and the statistician ensured that treatment codes remained blinded. The 


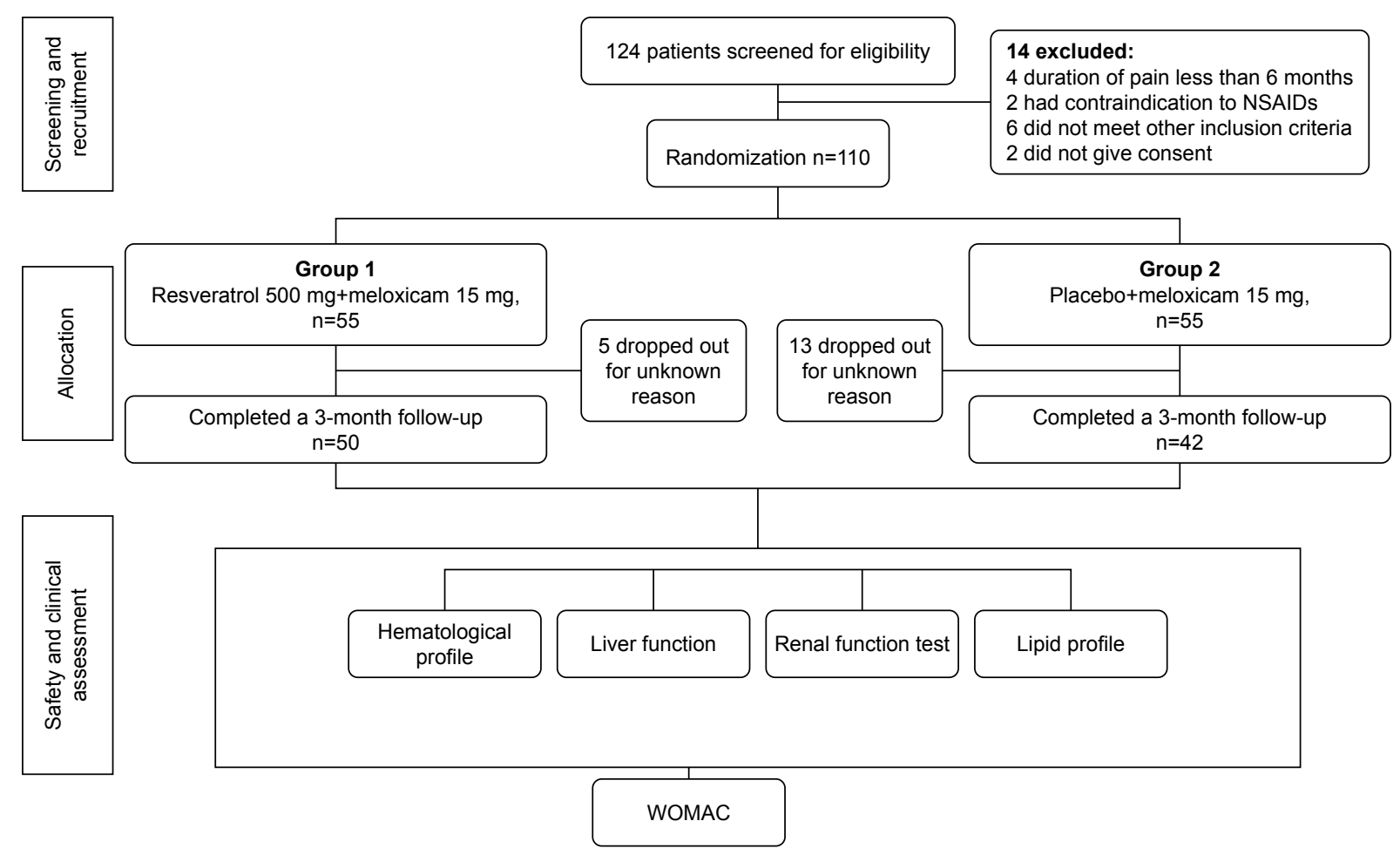

Figure I Flowchart of the study.

Note: n, number of subjects.

Abbreviations: NSAIDs, nonsteroidal anti-inflammatory drugs; WOMAC, Western Ontario and McMaster Universities Osteoarthritis Index.

participants were allocated to either placebo group $(n=55)$ or resveratrol group $(\mathrm{n}=55)$. In the resveratrol-treated group, the patients received $500 \mathrm{mg}$ resveratrol once daily, whereas the patients in the placebo group received a placebo formula that contains pregelatinized starch formulated as a capsule dosage form identical in shape, appearance, and color to that of resveratrol used in the test group. All participants in both groups received $15 \mathrm{mg}$ meloxicam (Mlx) once daily as a sustained therapy for their knee OA complaints during the entire trial period. Both groups were allowed to receive vitamin $\mathrm{D}$ within the recommended daily allowance. The basic characteristics of all participants were recorded in a standard questionnaire form. The study was carried out as a 3-month follow-up with the following four visits: at baseline day (Visit 1), day 30 (Visit 2), day 60 (Visit 3), and day 90 (Visit 4) of the treatment. Clinical assessment and physical examination were recorded at each visit while blood samples for hematological and clinical laboratory tests were obtained from each individual twice, at the baseline and after the end of the trial (day 90).

\section{Outcome measurement}

The primary outcome measure for this study was the assessment of lipid-hematological profile, liver and kidney functions, and a comprehensive short-term follow-up of the clinical and physical examinations, vital signs, body weight alteration, and occurrence of drug-related adverse events. All adverse events reported by the patients at each visit were recorded during each visit using a modified form of the NCI Common Terminology Criteria for Adverse Events version 4.0. Furthermore, serum vitamin $D$ level was measured for each patient twice in a pre- and postinterventional schedule to investigate the expected effect of resveratrol on the serum vitamin D level. ${ }^{25}$ The secondary outcome measure includes the evaluation of pain and disease functional activity using the patient global assessment of the change in WOMAC (Likert scale version; score range $0-96)^{26}$ at each visit during the trial period.

\section{Hematological and biochemical assessment}

Fasting blood sample $(8.0 \mathrm{~mL})$ was taken from each participant twice, at baseline and at the end of the treatment. Three milliliters of aliquot was collected in an EDTA tube and used for the determination of hemoglobin $(\mathrm{Hb})$ concentration, hematocrit (Hct\%), erythrocyte count (red blood cells), leukocyte count (white blood cells), and platelet count. The other $5.0 \mathrm{~mL}$ was collected in a plain tube for isolation of 
the serum by centrifugation at 5,000 rpm for 10 minutes and stored at $-70^{\circ} \mathrm{C}$ until the time of analysis. The serum was utilized for the assessment of the markers of liver function (serum glutamate pyruvate transaminase [GPT], glutamate oxaloacetate transaminase [GOT], and alkaline phosphatase [ALP] activities), renal function (urea and creatinine), lipid profile (total cholesterol, triglyceride [TG], high-density lipoprotein-cholesterol [HDL-c], and low-density lipoproteincholesterol [LDL-c]), and vitamin D levels at baseline and at the end of the treatment spectrophotometrically using the clinical chemistry analyzer Cobas c311 and ready-made kits (Hoffman-La Roche Ltd., Basel, Switzerland) according to the manufacturer's specifications.

\section{Assessment of functional activity and pain score}

Functional disability and pain were assessed by the investigators at baseline and on each follow-up visit (days 0 , 30, 60, and 90). Questionnaire-based assessment of pain, stiffness, and physical function was performed using the WOMAC. ${ }^{26}$

\section{Statistical analyses}

Detailed statistical analyses were performed using GraphPad Prism 5.0.1 software (GraphPad Software Inc., La Jolla, CA, USA). The baseline demographic data and patient characteristics were compared between the treatment groups. Categorical data were analyzed with the chi-squared test. Continuous variables were analyzed using independent sample $t$-test or the two-way ANOVA and Bonferroni's post hoc test. A $P$-value of less than 0.05 was considered to be statistically significant.

\section{Results}

Among the 124 knee OA patients screened for eligibility, only 110 patients were recruited in this study and 14 patients were excluded for various reasons. The qualified participants were randomly assigned to the resveratrol-treated group $(n=55)$ and the placebo group $(n=55)$. Only 92 patients completed the 90-day intervention and the follow-up investigations (Figure 1).

\section{Baseline characteristics of the patients}

The baseline characteristics of the participants are shown in Table 1. Mean age, body mass index (BMI), disease duration, the severity of pain and functional activity measured by WOMAC, and associated diseases in both the resveratroltreated group and the placebo group were comparable. Hence, at baseline, the demographic and clinical characteristics were
Table I Demographic data and baseline characteristics of the knee OA patients treated with Res coadministered with Mlx (Mlx+Res) or Mlx with placebo (Mlx+placebo)

\begin{tabular}{|c|c|c|c|}
\hline Variables & $\begin{array}{l}\text { Mlx+Res } \\
(n=50)\end{array}$ & $\begin{array}{l}\text { Mlx+placebo } \\
(n=42)\end{array}$ & $P$-value \\
\hline \multicolumn{4}{|l|}{ Gender, n (\%) } \\
\hline Male & $13(26)$ & $10(23.8)$ & 0.03 \\
\hline Female & $37(74)$ & $32(76.2)$ & 0.06 \\
\hline Age (years) & $58.2 \pm 9.1$ & $57.6 \pm 8.2$ & 0.77 \\
\hline Body weight (kg) & $80.5 \pm 16.1$ & $82.4 \pm 11.0$ & 0.55 \\
\hline BMI $\left(\mathrm{kg} / \mathrm{m}^{2}\right)$ & $30.7 \pm 5.6$ & $32.1 \pm 4.6$ & 0.1 \\
\hline Disease duration (years) & $3.5 \pm 3.2$ & $3.9 \pm 2.9$ & 0.45 \\
\hline \multicolumn{4}{|l|}{ Disease grade, n (\%) } \\
\hline Grade I & $7(14)$ & $5(12)$ & 0.12 \\
\hline Grade 2 & $25(50)$ & $18(42.8)$ & \\
\hline Grade 3 & $18(36)$ & $19(45.2)$ & \\
\hline $\begin{array}{l}\text { Baseline WOMAC total } \\
\text { score }(0-96)\end{array}$ & $59.6 \pm 13.7$ & $59.3 \pm 7.4$ & 0.2 \\
\hline \multicolumn{4}{|l|}{ Associated diseases, n (\%) } \\
\hline Hypertension & $20(40.0)$ & $18(42.8)$ & 0.10 \\
\hline Diabetes mellitus & $8(16.0)$ & $5(12)$ & 0.12 \\
\hline Smoking habits & $3(6.0)$ & $2(4.7)$ & 0.11 \\
\hline
\end{tabular}

Notes: Values are presented as either $\mathrm{n}(\%)$ or mean \pm SD. $n$, number of patients. Abbreviations: BMI, body mass index; Mlx, meloxicam; OA, osteoarthritis; Res, resveratrol; WOMAC, Western Ontario and McMaster Universities Osteoarthritis Index.

reasonably well balanced among the two groups except for gender $(P=0.03)$, which was not a potential variable in this study. Moreover, the baseline value of hematological tests, lipid profile, liver function test, and renal function test also demonstrated a nonsignificant difference, except for serum GPT (Figure 2B), where a significant difference was reported between both groups $(P<0.05)$.

\section{Hematological evaluation}

As a part of the safety evaluation, the hematological profile of the patients was evaluated to monitor any abnormal effects of resveratrol, when used as an adjuvant therapy with Mlx, on the complete blood picture. The values of the hematological markers remained within the normal range at baseline and on the last day of the treatment (day 90). These findings demonstrate the safety aspect of resveratrol on the hematology profile of the patients (Table 2).

\section{Body mass index}

In Table 3, the mean values of the BMI for the Mlx+Res group in the different timelines were comparable with those of baseline within the same group. Meanwhile, no significant differences were reported in the placebo+Mlx group $(P>0.05)$ during the treatment period. Similarly, no significant differences were reported between the Res+Mlx-treated group and the placebo+Mlx-treated group $(P>0.05)$. 

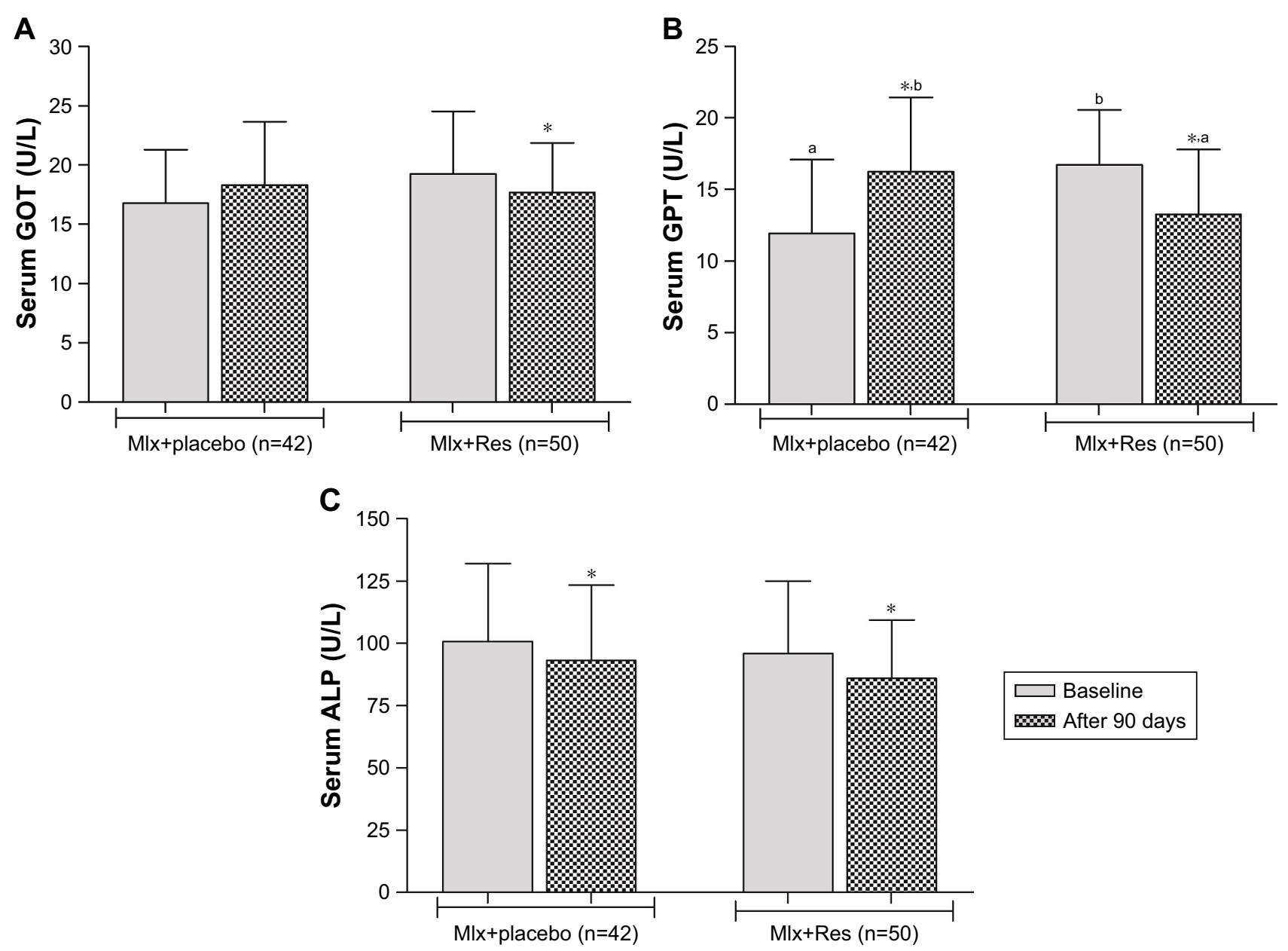

Figure 2 Effect of resveratrol coadministration with Mlx on (A) serum GOT, (B) serum GPT, and (C) serum ALP as markers of liver function in patients with mild to moderate knee OA.

Notes: *Significantly different compared with baseline in each group (paired $t$-test, $P<0.05$ ). Values with different superscript letters (a,b) are significantly different among the groups (ANOVA, $P<0.05)$. n, number of patients.

Abbreviations: ALP, alkaline phosphatase; GOT, glutamate oxaloacetate transaminase; GPT, glutamate pyruvate transaminase; Mlx, meloxicam; Res, resveratrol.

\section{Liver and kidney functions and lipid profile}

In the Mlx+Res group, the serum levels of GOT, GPT, and ALP were significantly decreased at day 90 compared with the corresponding baseline values $(P<0.05)$. Meanwhile, a significant elevation of these enzymes has been observed in the Mlx+placebo-treated group at day 90, except for ALP that displays a significant reduction in both groups (Figure 2A-C). Regarding the influence of resveratrol on the renal function, Figure $3 \mathrm{~A}$ and $\mathrm{B}$ shows a significant decrease in serum creatinine and serum urea levels of the Mlx+Res group compared with baseline values $(P<0.05)$; meanwhile, the serum

Table 2 Effect of coadministration of Res with Mlx and Mlx with placebo (Mlx+placebo) on the hematological parameters of patients with knee OA

\begin{tabular}{|c|c|c|c|c|}
\hline \multirow[t]{2}{*}{ Parameters } & \multicolumn{2}{|c|}{ Mlx+Res $(n=50)$} & \multicolumn{2}{|c|}{ Mlx+placebo $(n=42)$} \\
\hline & Baseline & After 90 days & Baseline & After 90 days \\
\hline $\mathrm{Hb}(\mathrm{g} / \mathrm{dL})$ & $13.2 \pm 1.5^{\mathrm{a}}$ & $13.2 \pm 1.4^{a}$ & $13.2 \pm 1.3^{\mathrm{a}}$ & $13.1 \pm 1.0^{\mathrm{a}}$ \\
\hline Hct (\%) & $40.3 \pm 4.3^{\mathrm{a}}$ & $40.0 \pm 4.0^{\mathrm{a}}$ & $40.7 \pm 3.8^{\mathrm{a}}$ & $40.0 \pm 3.1^{\mathrm{a}}$ \\
\hline $\mathrm{RBC}$ count $\times 10^{6}$ cells $/ \mu \mathrm{L}$ & $4.9 \pm 0.4^{\mathrm{a}}$ & $4.9 \pm 0.4^{a}$ & $5.0 \pm 0.5^{\mathrm{a}}$ & $4.9 \pm 0.4^{a}$ \\
\hline WBC count $\times 10^{3}$ cells $/ \mu \mathrm{L}$ & $6.9 \pm 1.5^{\mathrm{a}}$ & $6.8 \pm 2.1^{\mathrm{a}}$ & $7.2 \pm 1.8^{\mathrm{a}}$ & $6.9 \pm 1.5^{\mathrm{a}}$ \\
\hline Platelet count $\times 10^{9}$ cells $/ \mathrm{L}$ & $225 \pm 54^{\mathrm{a}}$ & $224 \pm 53^{a}$ & $25 I \pm 59^{a}$ & $227 \pm 54^{*, a}$ \\
\hline
\end{tabular}

Notes: Values are presented as mean $\pm S D$; *significantly different compared with baseline value in each group (paired $t$-test, $P<0.05)$; values with different superscripts (a,b) within each parameter are significantly different (ANOVA, $P<0.05$ ). $n$, number of patients.

Abbreviations: Hb, hemoglobin; Hct, hematocrit; Mlx, meloxicam; OA, osteoarthritis; RBC, red blood cell; Res, resveratrol; WBC, white blood cell. 
Table 3 Effect of coadministration of Res with Mlx and Mlx with placebo (Mlx+placebo) on the BMI of patients with knee OA

\begin{tabular}{|c|c|c|c|c|c|c|c|c|}
\hline \multirow[t]{2}{*}{ Parameter } & \multicolumn{4}{|c|}{ Mlx $+\operatorname{Res}(n=50)$} & \multicolumn{4}{|c|}{ Mlx+placebo $(n=42)$} \\
\hline & Day 0 & Day 30 & Day 60 & Day 90 & Day 0 & Day 30 & Day 60 & Day 90 \\
\hline BMI $\left(\mathrm{kg} / \mathrm{m}^{2}\right)$ & $30.5 \pm 5.7$ & $30.6 \pm 5.8$ & $30.6 \pm 5.8$ & $30.5 \pm 5.7$ & $32.9 \pm 4.5$ & $33.1 \pm 4.7$ & $33.1 \pm 4.9$ & $32.9 \pm 4.9$ \\
\hline
\end{tabular}

Notes: Values were presented as mean $\pm S D$; paired $t$-test: $P>0.05$; ANOVA: $P>0.05$. No significant difference reported between the two groups. $n$, number of patients. Abbreviations: BMI, body mass index; Mlx, meloxicam; OA, osteoarthritis; Res, resveratrol.

urea was significantly elevated in the Mlx+placebo-treated group after 90 days (Figure 3A). Moreover, no significant changes were reported in serum LDL-c and HDL-c levels in both groups after 90 days of treatment (Figure 4C and D). However, a statistically significant reduction was reported in the total cholesterol and TG levels of the Mlx+Res-treated group $(P<0.05)$ (Figure 4A and $\mathrm{B})$.

\section{Vitamin D level}

In Figure 5, no significant changes in the serum levels of vitamin D were detected in both groups compared with baseline values and between the two groups, both at baseline and after the end of the treatment $(P>0.05)$.

\section{Pain and functional disability outcome scores}

In Table 4, coadministration of resveratrol with Mlx significantly improves the total WOMAC score after 30 days compared with both baseline value and that of the placebo plus Mlx-treated group within the same period $(P<0.05)$. The effect of resveratrol continues to improve the WOMAC score after 60 and 90 days in a nonsignificant manner compared with its value after 30 days $(P>0.05)$. Additionally, significant improvement in WOMAC subscales including pain, stiffness, and physical function was reported in the resveratrol-treated group compared with the baseline value of the same group and the corresponding subscales in all follow-up visits in the placebo-treated group (Table 4).

\section{Adverse events' monitoring}

During the 90-day treatment period, no major adverse events were reported, and resveratrol was found to be well tolerated in all patients during the entire study period.

\section{Discussion}

The increasing demand for safe and effective alternative drugs for the treatment of OA can be addressed by identifying promising nutraceutical candidates. In this regard, resveratrol has demonstrated a great potential in preventing and/or slowing the breakdown of articular cartilage and extracellular matrix in the preclinical studies. ${ }^{15} \mathrm{In}$ human articular chondrocytes, resveratrol also offers antiapoptotic, anti-inflammatory, and antioxidant effects..$^{16,27}$ Similarly, another preclinical study demonstrated the potent anabolic and anti-catabolic potential of resveratrol in human adult articular chondrocytes via the inhibition of

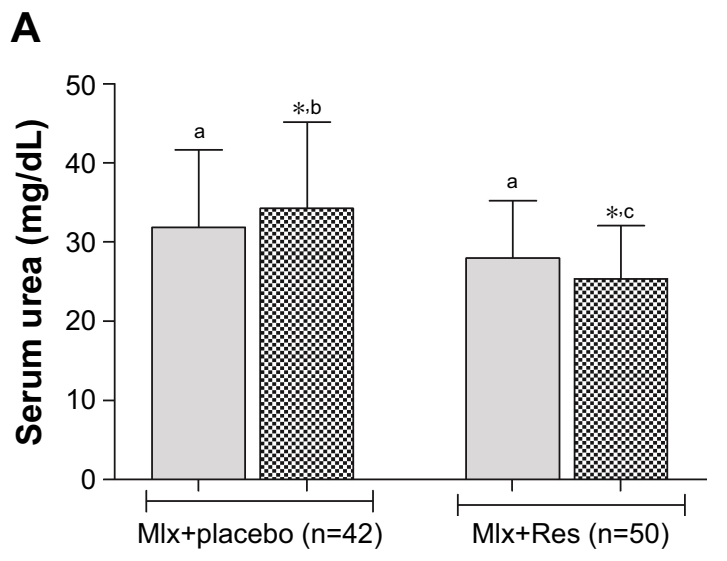

B

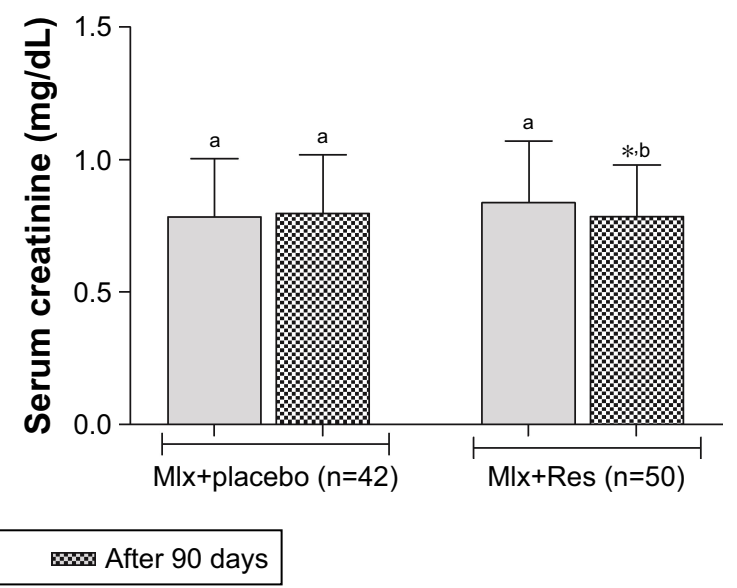

Figure 3 Effect of resveratrol coadministration with Mlx on serum levels of $(\mathbf{A})$ urea and $(\mathbf{B})$ creatinine in patients with mild to moderate knee OA. Notes: *Significantly different compared with the baseline in each group (paired $t$-test, $P<0.05$ ); values with non-identical letters (a-c) are significantly different among groups (ANOVA, $P<0.05$ ). $n$, number of patients.

Abbreviations: Mlx, meloxicam; OA, osteoarthritis; Res, resveratrol. 

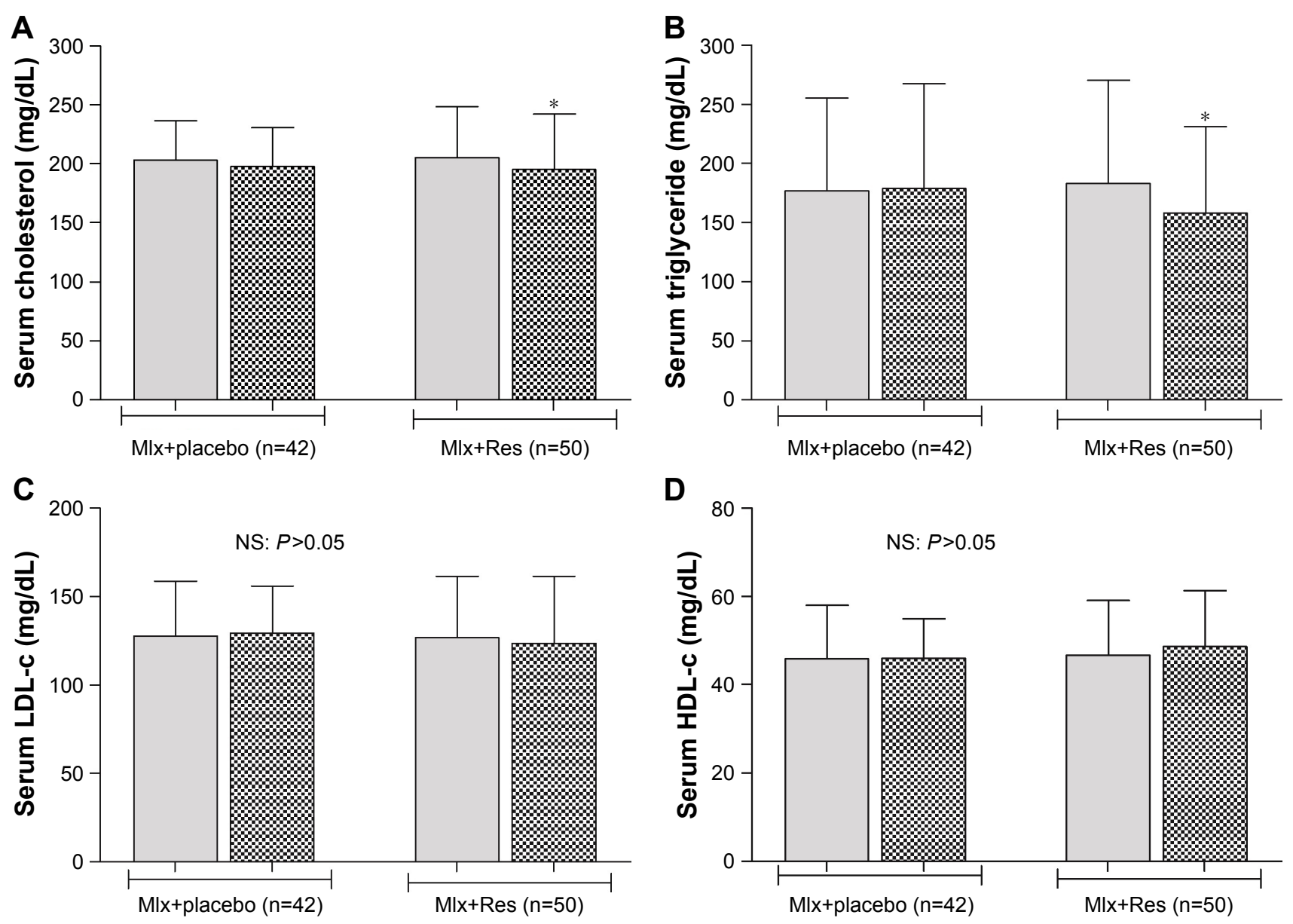

$\square$ Baseline $\quad \mathbf{5 0}$ After 90 days

Figure 4 Effect of resveratrol coadministration with Mlx on the serum levels of (A) cholesterol, (B) TG, (C) LDL-c, and (D) HDL-c in patients with mild to moderate knee OA.

Notes: *Significantly different compared with baseline in each group (paired $t$-test, $P<0.05$ ). $n$, number of patients.

Abbreviations: HDL-c, high-density lipoprotein-cholesterol; LDL-c, low-density lipoprotein-cholesterol; Mlx, meloxicam; NS, not significant; OA, osteoarthritis; Res, resveratrol; TG, triglyceride.

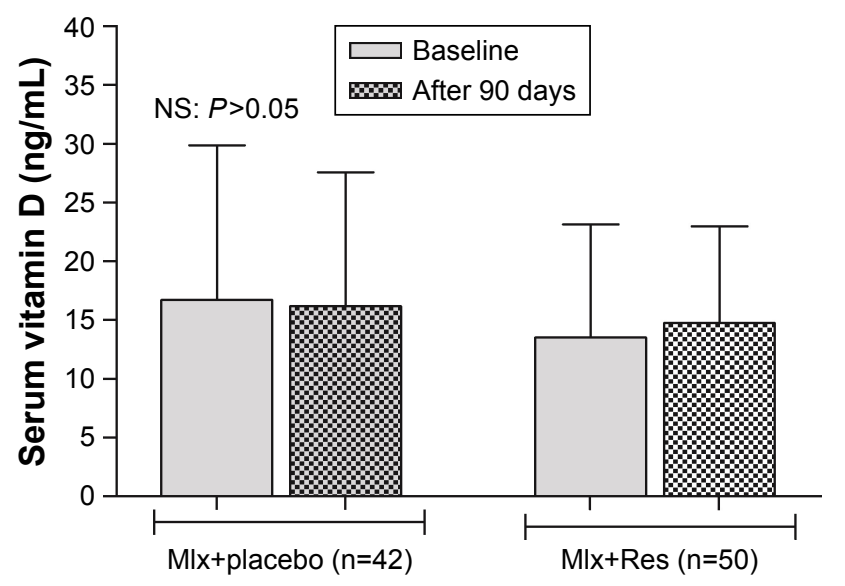

Figure 5 Effect of resveratrol coadministration with Mlx on the serum level of vitamin $D$ in patients with mild to moderate knee OA.

Notes: Paired $t$-test: $P>0.05$; ANOVA: $P>0.05$. n, number of patients.

Abbreviations: Mlx, meloxicam; NS, not significant; OA, osteoarthritis; Res, resveratrol. the matrix-degrading enzyme. ${ }^{28}$ In light of these promising preclinical findings and previously reported evidence, resveratrol appears to be a potential anti-osteoarthritic agent. Despite the large number of preclinical studies conducted on the efficacy of natural supplements, such as resveratrol, silibinin, and many other nutraceuticals, in the musculoskeletal disorders, to our knowledge the clinical trials performed on the use of these natural products in OA are scarce. This study will be the first one that investigates the potential of therapeutic use and safety of resveratrol in the management of knee OA. This is based on a short-term follow-up of a group of patients utilizing the functional assessment of pain and disability index, the WOMAC scale, which is a well established and validated scale for the evaluation of pain and disability as manifestations of knee OA. In this study, the total WOMAC 
Table 4 Effect of coadministration of Res with Mlx (Mlx+Res) on the WOMAC subscales and total WOMAC in patients with mild to moderate knee $O A$

\begin{tabular}{|c|c|c|c|c|c|c|c|c|}
\hline \multirow[t]{2}{*}{ WOMAC area } & \multicolumn{4}{|c|}{ Mlx+Res $(n=50)$} & \multicolumn{4}{|c|}{ Mlx+placebo $(n=42)$} \\
\hline & Baseline & 30 days & 60 days & 90 days & Baseline & 30 days & 60 days & 90 days \\
\hline Stiffness (0-8) & $4.3 \pm 2.2^{*, a}$ & $1.1 \pm 1.3^{*, b}$ & $0.8 \pm 1.3^{*, b}$ & $0.7 \pm 1.2^{*, b}$ & $5.8 \pm 1.4^{\mathrm{a}}$ & $5.3 \pm 1.8^{b}$ & $5.5 \pm 1.9^{b}$ & $4.6 \pm 2.3^{b}$ \\
\hline Pain $(0-20)$ & $13.4 \pm 3.3^{\mathrm{a}}$ & $3.6 \pm 3.1^{*, \mathrm{~b}}$ & 2. $1 \pm 2.6^{*, b}$ & $1.8 \pm 2.5^{*, \mathrm{~b}}$ & $13.8 \pm 2.4^{a}$ & $12.1 \pm 4.0^{\mathrm{a}}$ & $12.2 \pm 4.0^{\mathrm{a}}$ & $11.9 \pm 4.4^{\mathrm{a}}$ \\
\hline Physical function (0-68) & $41.8 \pm 9.3^{\mathrm{a}}$ & $|1.3 \pm 11 .|^{*, b}$ & $7.2 \pm 7.4^{*, \mathrm{~b}}$ & $5.9 \pm 5.4^{*, b}$ & $44.1 \pm 8.3^{\mathrm{a}}$ & $41.1 \pm 16.2^{\mathrm{a}}$ & $39.6 \pm 11.6^{\mathrm{a}}$ & $37 \pm 12^{\mathrm{a}}$ \\
\hline Total WOMAC score (0-96) & $59.6 \pm 13.7^{a}$ & $16.2 \pm 14.6^{*, \mathrm{~b}}$ & $9.5 \pm 9.8^{*, c}$ & $8.1 \pm 8.2 * c$ & $63.7 \pm 11.1^{\mathrm{a}}$ & $58.6 \pm 15.5^{\mathrm{a}}$ & $59 \pm 14.4^{\mathrm{a}}$ & $56 \pm 16^{a}$ \\
\hline
\end{tabular}

Notes: Values are presented as mean \pm SD; $*$ significantly different compared with baseline values in each group (paired $t$-test, $P<0.05)$. Values with different superscripts $(\mathrm{a}-\mathrm{c})$ within each parameter are significantly different (ANOVA, $P<0.05$ ). $n$, number of patients.

Abbreviations: Mlx, meloxicam; OA, osteoarthritis; Res, resveratrol; WOMAC, Western Ontario and McMaster Universities Osteoarthritis Index.

scores were significantly lower in the patients treated with resveratrol and Mlx than in those treated with placebo plus Mlx in a 3-month interval. We also found an overall clinical improvement by resveratrol of all the three subscales of the WOMAC score (pain, stiffness, and physical function) in the resveratrol-treated group compared with the placebo-treated patients. This result was in concurrence with the finding of many relevant preclinical studies that demonstrate the analgesic activity of resveratrol in experimental models of OA. ${ }^{14,29-31}$ More recently, the adjuvant use of resveratrol with the conventional antirheumatic drugs exhibited a profound benefit in the improvement of the clinical and biochemical markers of rheumatoid arthritis. ${ }^{32}$ Moreover, apart from the reported clinical improvement in this study, a significant reduction in the disease-related inflammatory biomarkers including IL-6, IL-1 $\beta$, and TNF- $\alpha$ has been observed (unpublished data). These results suggest that resveratrol could be a potential analgesic that decreases the pain and discomfort of knee OA and improves the patient's general condition and quality of daily life. The exact mechanism behind the analgesic effect of resveratrol is not fully evaluated; however, it is believed that cumulative multifactorial functions, including chondroprotective, analgesic, and anti-inflammatory activities, could play a role. Moreover, its ability to reduce cartilage destruction and loss of matrix proteoglycan content in the cartilage could be attributed to the decrease in the apoptosis rate of the chondrocytes and the level of NO in the synovial fluid. ${ }^{4}$ In addition, the recently reported reduction in the expression of COX-2 and PGE2 in the rat adjuvant arthritis model ${ }^{33}$ might be a convincing mechanism for resveratrol's pain-relieving action. Similar to most polyphenols, we cannot rule out the intrinsic anti-oxidant capacity of resveratrol, which may contribute to its anti-osteoarthritic effect. Resveratrol also induces the expression of a number of anti-oxidant enzymes, making it difficult to interpret the precise contribution of each mechanism to the overall reduction in pain intensity of the participants in this study. The effectiveness of herbal medicine and polyphenol-containing compounds is reported in many clinical studies, which are classified as the most popular type of complementary and alternative medical options for the treatment of $\mathrm{OA}, 34,35$ and the safety of these agents has been investigated in numerous studies. The patients in the resveratrol-treated group were administered $500 \mathrm{mg}$ resveratrol/day for 3 months. To ensure that resveratrol supplementation does not cause adverse effects, we monitor the patients for the emergence of hematological, hepatic, and renal adverse effects. Additionally, the regular clinical examination was performed for each patient to report any deleterious effect due to the orally administered resveratrol. No major adverse effects were recorded, and this result may be considered as additional evidence that supports the safety and tolerability of resveratrol at the given dose. The outcomes of this study will help to determine the efficacy of a short-term treatment with resveratrol in knee OA patients. The results also provide an extension and confirmation to the previous clinical observations conducted in the healthy volunteers or cancerous individuals that assessed the safety of resveratrol utilizing higher and lower doses during different periods. Taken together, the findings of these studies suggest that adverse reactions to resveratrol at doses of less than $1,000 \mathrm{mg} /$ day are uncommon or mild, and short-term resveratrol supplementation is well tolerated. Our findings are in tune with many previously reported data, where a daily dose of less than $1,000 \mathrm{mg}$ /day has been used. ${ }^{20,36,37}$ In this study, renal function tests demonstrated a significant reduction in the level of serum creatinine and urea in the Mlx+Res group, which may be attributed to the nephroprotective effect of resveratrol previously reported in the cisplatin induced-kidney injury model..$^{38}$ Moreover, the present study revealed that resveratrol did not negatively impact the liver function. This finding was in tune with the study that defines resveratrol as a safe supplement with negligible harmful effects. ${ }^{39}$ Furthermore, the hepatoprotective role of resveratrol is reported in different models of hepatitis that 
linked this action with its antioxidant property. In this study, no significant alteration has been observed in the lipid profile of both groups, except total cholesterol and TG levels that display a significant reduction in the resveratrol-treated group. Furthermore, resveratrol did not change the hematological markers of the patients, and this result came in tune with previous studies that reported hematological safety in humans and experimental animals. ${ }^{40,41}$ In this study, the daily consumption of $500 \mathrm{mg}$ /day resveratrol did not affect the $\mathrm{BMI}$ of the patients, and this result was consistent with that reported by other studies. ${ }^{39,42}$ A previous study has hypothesized that resveratrol and vitamin $\mathrm{D}$ demonstrate potential interactions that could seriously affect the clinical outcome of each other. ${ }^{25}$ In this study, the patients were allowed to consume vitamin D within the daily recommended amount; however, no changes were reported in the serum levels of vitamin $\mathrm{D}$ at the end of the treatment. This study encountered many limitations related to the small sample size $(n=110)$ and short duration of treatment (90 days). The absence of a dose-response model to assess the effects at low dose against a high dose of resveratrol is another limitation of this study. Also, because of the relatively short duration of follow-up, we did not assess the radiological outcomes at the end of the trial. Meanwhile, the strong point of this study is that it is a double-blind randomized placebo-controlled study, which includes measurement of both the efficacy and safety of resveratrol that provides convincing evidence for an optimal therapeutic application of resveratrol in a musculoskeletal disorder such as knee OA. The use of rescue medication, which is not allowed in this study, may be prone to bias. However, the similarity of the type and amount of pain medication (Mlx) used between the two groups makes this unlikely.

\section{Conclusion}

The use of resveratrol as an "add-on" medication with Mlx was superior in terms of safety and efficacy to Mlx alone for the treatment of pain and improvement of physical function in patients with knee OA.

\section{Acknowledgments}

The presented data were abstracted from a Doctor of Philosophy thesis $(\mathrm{PhD})$ submitted by Marouf BH to the Department of Pharmacology, College of Medicine, University of Sulaimani. ${ }^{43}$ The project as a whole (including the experiments, preparation of the thesis, and the publication of data abstracted from the thesis) was financially supported and ethically approved by the University of Sulaimani (Certificate
ID: 42 on 21/11/2016). The authors gratefully thank the kind technical supports from the Shar Teaching Hospital, General Teaching Hospital, and the Rheumatology and Physical Rehabilitation Center in Sulaimani.

\section{Disclosure}

The authors report no conflicts of interest in this work.

\section{References}

1. Jiménez G, Cobo-Molinos J, Antich C, López-Ruiz E. Osteoarthritis: Trauma vs disease. Adv Exp Med Biol. 2018;1059:63-83.

2. Berenbaum F. Osteoarthritis as an inflammatory disease osteoarthritis is not osteoarthrosis! Osteoarthritis Cartilage. 2013;21(1):16-21.

3. Rahmati M, Mobasheri A, Mozafari M. Inflammatory mediators in osteoarthritis: a critical review of the state-of-the-art, current prospects, and future challenges. Bone. 2016;85:81-90.

4. Wang J, Gao JS, Chen JW, Li F, Tian J. Effect of resveratrol on cartilage protection and apoptosis inhibition in experimental osteoarthritis of rabbit. Rheumatol Int. 2012;32(6):1541-1548.

5. Choudhary D, Kothari P, Tripathi AK, et al. Spinacia oleracea extract attenuates disease progression and sub-chondral bone changes in monosodium iodoacetate-induced osteoarthritis in rats. BMC Complement Altern Med. 2018;18(1):69.

6. Liu X, Machado GC, Eyles JP, Ravi V, Hunter DJ. Dietary supplements for treating osteoarthritis: a systematic review and meta-analysis. $\mathrm{Br} J$ Sports Med. 2018;52(3):167-175.

7. Castrogiovanni P, Trovato FM, Loreto C, Nsir H, Szychlinska MA, Musumeci G. Nutraceutical supplements in the management and prevention of osteoarthritis. Int J Mol Sci. 2016;17(12):E2042.

8. Dybkowska E, Sadowska A, Świderski F, Rakowska R, Wysocka K The occurrence of resveratrol in foodstuffs and its potential for supporting cancer prevention and treatment. A review. Rocz Panstw Zakl Hig. 2018;69(1):5-14.

9. Kuo HP, Wang R, Lin YS, Lai JT, Lo YC, Huang ST. Pilot scale repeated fed-batch fermentation processes of the wine yeast Dekkera bruxellensis for mass production of resveratrol from Polygonum cuspidatum. Bioresour Technol. 2017;243:986-993.

10. Karimi Dermani F, Saidijam M, Amini R, Mahdavinezhad A, Heydari K, Najafi R. Resveratrol Inhibits proliferation, invasion, and epithelialmesenchymal transition by increasing miR-200c expression in HCT-116 colorectal cancer cells. J Cell Biochem. 2017;118(6):1547-1555.

11. Limmongkon A, Janhom P, Amthong A, et al. Antioxidant activity, total phenolic, and resveratrol content in five cultivars of peanut sprouts. Asian Pac J Trop Biomed. 2017;7(4):332-338.

12. Sadeghi A, Seyyed Ebrahimi SS, Golestani A, Meshkani R. Resveratrol ameliorates palmitate-induced inflammation in skeletal muscle cells by attenuating oxidative stress and $\mathrm{JNK} / \mathrm{NF}-\kappa \mathrm{B}$ pathway in a SIRT1-independent mechanism. J Cell Biochem. 2017;118(9): 2654-2663.

13. Pan X, Chen J, Wang W, et al. Resveratrol-induced antinociception is involved in calcium channels and calcium/caffeine-sensitive pools. Oncotarget. 2017;8(6):9399-9409.

14. Wang G, Hu Z, Song X, et al. Analgesic and anti-inflammatory activities of Resveratrol through classic models in mice and rats. Evid Based Complement Alternat Med. 2017;2017:9, 5197567.

15. Jiang M, Li X, Yu X, et al. Oral administration of Resveratrol alleviates osteoarthritis pathology in C57BL/6J mice model induced by a high-fat diet. Mediators Inflamm. 2017;2017:11, 7659023.

16. Nguyen C, Savouret JF, Widerak M, Corvol MT, Rannou F, Corvol JF, Resveratrol RF. Resveratrol, potential therapeutic interest in joint disorders: a critical narrative review. Nutrients. 2017;9(1):E45.

17. Dave M, Attur M, Palmer G, et al. The antioxidant resveratrol protects against chondrocyte apoptosis via effects on mitochondrial polarization and ATP production. Arthritis Rheum. 2008;58(9):2786-2797. 
18. Shakibaei M, Csaki C, Nebrich S, Mobasheri A. Resveratrol suppresses interleukin-1beta-induced inflammatory signaling and apoptosis in human articular chondrocytes: potential for use as a novel nutraceutical for the treatment of osteoarthritis. Biochem Pharmacol. 2008;76(11):1426-1439.

19. Kutil Z, Temml V, Maghradze D, et al. Impact of wines and wine constituents on cyclooxygenase-1, cyclooxygenase-2, and 5-lipoxygenase catalytic activity. Mediators Inflamm. 2014;2014:8, 178931.

20. Anton SD, Embry C, Marsiske M, et al. Safety and metabolic outcomes of resveratrol supplementation in older adults: results of a twelve-week, placebo-controlled pilot study. Exp Gerontol. 2014;57:181-187.

21. Riccioni G, Gammone MA, Currenti W, D’Orazio N. Effectiveness and safety of dietetic supplementation of a new nutraceutical on lipid profile and serum inflammation biomarkers in hypercholesterolemic patients. Molecules. 2018;23(5):1168.

22. Hochberg MC, Altman RD, April KT, et al. American College of Rheumatology 2012 recommendations for the use of nonpharmacologic and pharmacologic therapies in osteoarthritis of the hand, hip, and knee. Arthritis Care Res. 2012;64(4):465-474.

23. Kellgren JH, Lawrence JS. Radiological assessment of osteo-arthrosis. Ann Rheum Dis. 1957;16(4):494-502.

24. Wu CW, Morrell MR, Heinze E, et al. Validation of American College of Rheumatology classification criteria for knee osteoarthritis using arthroscopically defined cartilage damage scores. Semin Arthritis Rheum. 2005;35(3):197-201.

25. Hayes DP. Resveratrol and vitamin D: significant potential interpretative problems arising from their mutual processes, interactions and effects. Med Hypotheses. 2011;77(5):765-772.

26. Bellamy N, Buchanan WW, Goldsmith CH, Campbell J, Stitt LW. Validation study of WOMAC: a health status instrument for measuring clinically important patient relevant outcomes to antirheumatic drug therapy in patients with osteoarthritis of the hip or knee. J Rheumatol. 1988;15(12):1833-1840.

27. Ma C, Wang Y, Dong L, Li M, Cai W. Anti-inflammatory effect of resveratrol through the suppression of NF- $\mathrm{KB}$ and JAK/STAT signaling pathways. Acta Biochim Biophys Sin. 2015;47(3):207-213.

28. Im HJ, Li X, Chen D, et al. Biological effects of the plant-derived polyphenol resveratrol in human articular cartilage and chondrosarcoma cells. J Cell Physiol. 2012;227(10):3488-3497.

29. Wang ZM, Chen YC, Wang DP, Resveratrol WDP. Resveratrol, a natural antioxidant, protects monosodium iodoacetate-induced osteoarthritic pain in rats. Biomed Pharmacother. 2016;83:763-770.

30. Bazzo KO, Souto AA, Lopes TG, et al. Evidence for the analgesic activity of resveratrol in acute models of nociception in mice. $J$ Nat Prod. 2013;76(1):13-21.
31. Falchi M, Bertelli A, Galazzo R, Viganò P, Dib B. Central antalgic activity of resveratrol. Arch Ital Biol. 2010;148(4):389-396.

32. Khojah HM, Ahmed S, Abdel-Rahman MS, Elhakeim EH. Resveratrol as an effective adjuvant therapy in the management of rheumatoid arthritis: a clinical study. Clin Rheumatol. 2018;37(8):2035-2042.

33. Chen X, Lu J, An M, Ma Z, Zong H, Yang J. Anti-inflammatory effect of resveratrol on adjuvant arthritis rats with abnormal immunological function via the reduction of cyclooxygenase-2 and prostaglandin E2. Mol Med Rep. 2014;9(6):2592-2598.

34. Hussain SA, Jassim NA, Numan IT, Al-Khalifa II, Abdullah TA. Antiinflammatory activity of silymarin in patients with knee osteoarthritis. A comparative study with piroxicam and meloxicam. Saudi Med J. 2009;30(1):179-184.

35. Hussain SA, Mortada AH, Jasim NA, Gorial FI. Silibinin improves the effects of methotrexate in patients with Active Rheumatoid Arthritis: Pilot Clinical Study. Oman Med J. 2016;31(4):263-269.

36. Almeida L, Vaz-da-Silva M, Falcão A, et al. Pharmacokinetic and safety profile of trans-resveratrol in a rising multiple-dose study in healthy volunteers. Mol Nutr Food Res. 2009;53 Suppl 1(S1):S7-S15.

37. Patel KR, Brown VA, Jones DJ, et al. Clinical pharmacology of resveratrol and its metabolites in colorectal cancer patients. Cancer Res. 2010;70(19):7392-7399.

38. Hao Q, Xiao X, Zhen J, et al. Resveratrol attenuates acute kidney injury by inhibiting death receptor-mediated apoptotic pathways in a cisplatin-induced rat model. Mol Med Rep. 2016;14(4):3683-3689.

39. Juan ME, Vinardell MP, Planas JM. The daily oral administration of high doses of trans-resveratrol to rats for 28 days is not harmful. J Nutr. 2002;132(2):257-260.

40. Timmers S, Konings E, Bilet L, et al. Calorie restriction-like effects of 30 days of resveratrol supplementation on energy metabolism and metabolic profile in obese humans. Cell Metab. 2011;14(5):612-622.

41. Atmaca N, Yıldırım E, Güner B, Kabakçı R, Bilmen FS. Effect of resveratrol on hematological and biochemical alterations in rats exposed to fluoride. Biomed Res Int. 2014;2014:1-5.

42. Turner RT, Evans GL, Zhang M, Maran A, Sibonga JD. Is resveratrol an estrogen agonist in growing rats? Endocrinology. 1999;140(1): 50-54.

43. Marouf BH. Adjuvant use of resveratrol in patients with knee osteoarthritis: Comparative study with meloxicam [doctorate thesis]. Sulaimani: University of Sulaimani; 2018.
Clinical Interventions in Aging

\section{Publish your work in this journal}

Clinical Interventions in Aging is an international, peer-reviewed journal focusing on evidence-based reports on the value or lack thereof of treatments intended to prevent or delay the onset of maladaptive correlates of aging in human beings. This journal is indexed on PubMed Central, MedLine,

\section{Dovepress}

CAS, Scopus and the Elsevier Bibliographic databases. The manuscript management system is completely online and includes a very quick and fair peer-review system, which is all easy to use. Visit http://www.dovepress. com/testimonials.php to read real quotes from published authors. 